\title{
Link of the Hipparcos Stellar Reference Frame to the Distant Quasars
}

J.-F. Lestrade ${ }^{1}$, R.A. Preston ${ }^{2}$, D.L. Jones ${ }^{2}$, R.B. Phillips ${ }^{3}$, A.E.E. Rogers ${ }^{3}$, M.A. Titus ${ }^{3}$, J.E. Reynolds ${ }^{4}$, D.J. Jauncey ${ }^{4}$, D.C. Gabuzda ${ }^{5}$, M.J. Rioja ${ }^{6}$, J.C. Guirado ${ }^{7}$, P.M. McCulloch ${ }^{8}$

Abstract. The link of the Hipparcos and VLBI extragalactic reference frames has been achieved with a precision of $0.0005^{\prime \prime}$ in global orientation at the epoch of the catalogue (1991.25) and of $0.0003^{\prime \prime} / \mathrm{yr}$ in rate of rotation by VLBI observations of 12 radio-emitting stars.

Optical astrometric catalogues, i. e. stellar reference frames, have played a fundamental role in astronomy for galactic and solar system dynamical studies and for establishing the distance scale in the Universe. The ESA Hipparcos satellite launched in 1989 (Perryman 1989) completed a very successful mission in collecting data to produce an astrometric catalogue of 120000 stars with a mean precision of 0.001 " for their positions, annual proper motions and trigonometric parallaxes. It was envisioned early in the Hipparcos project that this new intrinsically high-precision catalogue should be linked to the inertial reference frame of the distant radio quasars, also very precise. This link would stop the global residual rate of rotation of the Hipparcos catalogue and would bring the radio and optical coordinate systems into coincidence. The alignment of these two coordinates systems facilitates the registration of optical and radio images, as already shown in Reynolds et al. (1995).

This link was achieved by accurately measuring the VLBI positions and proper motions of 12 radio-emitting stars relative to angularly nearby radio quasars which are part of the International Celestial Reference Frame (ICRF) made of the VLBI coordinates of 601 extragalactic radio sources (Arias et al. 1995). The comparison of the positions and proper motions measured for these 12 stars by both VLBI and Hipparcos yielded the 6 link parameters ( 3 Euler rotation angles and their 3 rates) as described in Lestrade et al. (1995). These 6 parameters determine the global orientation and the residual rate of rotation of the Hipparcos celestial sphere relative to the distant quasars.

We started a VLBI astrometry program for these 12 link stars in 1982 and used the Global VLBI network, the Deep Space Network, the EVN and the Australian VLBI network. We made repeated phase-referenced VLBI observations of these faint stellar radio sources to determine their astrometric parameters (position, annual proper motion and trigonometric parallax). The astrometric precision achieved by our program is better than $0.001^{\prime \prime}$ in general and the best precision is 100 microarcseconds $\left(\sigma^{2} \mathrm{CrB}\right)$ as shown in Table 1 . This precision is unprecedented in stellar astrometry and surpasses the Hipparcos result itself for this limited number of stars. The precision of the 6 parameters of the VLBI link provided to the Hipparcos Project in February 1996 is formally 0.5 milliarc-

\footnotetext{
${ }^{1}$ Observatoire de Paris-Meudon, F92195, Meudon, France.

2 Jet Propulsion Laboratory/Caltech, Pasadena, 91109, CA, USA. ${ }^{3}$ Haystack Observatory/MIT, Westford, 01886, Mass, USA. ${ }^{4}$ Australian Telescope, Epping, New South Wales 2121, Australia. ${ }^{5}$ Astro Space Center, Lebedev Institute, 117924, Moscow, Russia. ${ }^{6}$ JIVE, 7990 Dwingeloo, The Netherlands. ${ }^{7}$ Departamento de Astronomía, Universitat de València, 46100 Burjassot, València, Spain. ${ }^{8}$ University of Tasmania, Hobart, Tasmania, 7001, Australia.
} 
second for the global orientation at the epoch of the catalogue (1991.25) and is 0.3 milliarcsecond per year for the residual rate of rotation. The final link was done by combining results from different techniques (VLBI, MERLIN, VLA, Hubble Space Telescope, etc) in Kovalevksy et al. (1997) with a precision of 0.6 milliarcsecond in orientation and 0.25 milliarcsecond per year in rate. This final solution appears to be significantly weighted towards the VLBI solution.

In addition, the VLBI trigonometric parallaxes of the 12 stars of our VLBI program has provided an independent verification of the revised distance scale of the Universe brought by the Hipparcos catalogue. This important revision has solved one of the long-standing problem in cosmology; the age of the Universe is now consistent with the age of the oldest stars in globular clusters.

Our VLBI measurements are based on phase-referenced VLBI observations of radio-emitting stars that are faint source with millijansky flux density. The data were analyzed with the software SPRINT (Software for Phase-Reference INTerferometry) developed for high-precision astrometry of weak radio sources and described in Lestrade et al. (1990). This VLBI astrometric technique is precise enough to detect the wobble caused by the gravitational tug of possible extra-solar planets orbiting nearby radio-emitting stars. Some of us have started a new VLBI astrometric program to conduct this investigation on several radioemitting stars.

Table 1. Precisions of the VLBI astrometric parameters of the 12 link stars. The precision of each absolute coordinate combines the uncertainties of the relative position and of the reference quasar that is somewhat large for a few cases.

\begin{tabular}{ccccccc}
\hline \hline Name & $\begin{array}{c}\text { Absolute } \\
\text { coord. } \\
\text { (mas) }\end{array}$ & $\begin{array}{c}\text { Relative } \\
\text { coord. } \\
\text { (mas) }\end{array}$ & $\begin{array}{c}\text { Proper } \\
\text { motion } \\
\text { (mas/yr) }\end{array}$ & $\begin{array}{c}\text { Trigonometric } \\
\text { parallax } \\
\text { (mas) }\end{array}$ & $\begin{array}{c}\text { Post-fit } \\
\text { rms } \\
\text { (mas) }\end{array}$ & $\begin{array}{c}\text { Nber } \\
\text { degree } \\
\text { of Freed. }\end{array}$ \\
\hline LSI61303 & 1.24 & 0.56 & 0.32 & 0.38 & 0.75 & 9 \\
Algol & 0.64 & 0.52 & 0.18 & 0.73 & 1.24 & 21 \\
UXAri & 0.48 & 0.36 & 0.20 & 0.39 & 0.83 & 13 \\
HR1099 & 0.48 & 0.40 & 0.31 & 0.47 & 0.84 & 11 \\
HD283447 & $\mathbf{1 . 1 0}$ & 0.20 & 0.28 & 0.25 & 0.57 & 13 \\
HD32918 & $\mathbf{1 . 5 0}$ & 0.70 & 1.0 & 0.80 & 0.72 & 3 \\
HR5110 & 0.67 & 0.45 & 0.16 & 0.45 & 1.38 & 25 \\
$\sigma^{2}$ CrB & 0.29 & 0.12 & 0.05 & 0.10 & 0.32 & 23 \\
Cyg X1 & 0.94 & 0.32 & 0.14 & 0.30 & 0.59 & 7 \\
HD199178 & 1.95 & 0.26 & 0.43 & 0.33 & 0.46 & 7 \\
AR Lac & 0.94 & 0.36 & 0.19 & 0.37 & 0.66 & 9 \\
IM Peg & 0.69 & 0.39 & 0.47 & 0.68 & 0.64 & 5 \\
\hline
\end{tabular}

\section{References}

Arias et al. 1995. $A \& A, 303,604-608$.

Kovalevsky, J. et al. 1997. $A \& A, 332,620-633$.

Lestrade et al. 1990. $A J, 99,1663-1673$.

Lestrade et al. 1995. $A \mathcal{E} A$, 304, 182-188.

Perryman et al. 1989. Nature, 340, 111-116.

Reynolds et al. 1995. $A G A, 304,116-120$. 